\title{
DIFFUSIVE WAVES IN INHOMOGENEOUS MEDIA
}

\author{
by PAUL C. FIFE
}

(Received 17th March 1988)

\section{Introduction}

When the function $f(u)$ is of "bistable type", i.e. has two zeros $h_{-}$and $h_{+}$at which $f^{\prime}$ is negative and (for simplicity) has only one other zero between them, then the constant functions $u=h_{ \pm}$are $L_{\infty}$-stable solutions of the nonlinear diffusion equation

$$
u_{t}=u_{x x}+f(u)
$$

In addition, there are travelling wave solutions $u_{+}(x, t)$ and $u_{-}(x, t)$ which, if

$$
\int_{h_{-}}^{h_{+}} f(u) d s<0
$$

connect $h_{+}$to $h_{-}$in the sense that

$$
\lim _{t \rightarrow-\infty} u_{ \pm}(x, t)=h_{+} ; \lim _{t \rightarrow+\infty} u_{ \pm}(x, t)=h_{-},
$$

the convergence being uniform on bounded $x$-intervals. These solutions are of the form

$$
u_{ \pm}(x, t)=U( \pm x-c t)
$$

where $U(z)$ is a monotone function (the wave's profile), $U( \pm \infty)=h_{ \pm}$, and the velocity $c$ is a specific positive number depending on the function $f$.

Although $h_{+}$is stable in $L_{\infty}$, it is therefore unstable in the weaker topology of uniform convergence on bounded intervals. Besides these two connections between $h_{+}$ and $h_{-}$, there are others as well, characterized as pairs of travelling fronts approaching each other. If the sense of the inequality in (1.2) is reversed, then the roles of $h_{+}$and $h_{-}$ are interchanged.

For definiteness, take the intermediate zero of $f$ to be at the origin, so $f\left(h_{-}\right)=f(0)=$ $f\left(h_{+}\right)=0, h_{-}<0<h_{+}$.

A second well-known fact [2] about the travelling wave solutions (1.4) is that they attract a wide class of functions, exponentially in the $L_{\infty}$ norm. For example, let $S$ be the set of continuous bounded functions $\phi(x)$ which change sign exactly once, from 
negative to positive, and are bounded away from zero for large $|x|$. If $u(x, 0)=\phi(x) \in S$ and $u$ satisfies (1.1), then for some constants $M, m$, and $t_{0}$,

$$
\left|u(x, t)-u_{+}\left(x, t-t_{0}\right)\right| \leqq M e^{-m t}
$$

for all $x$ and positive $t$. This is in fact true without the restriction that $\phi$ have only one zero, provided $x \phi(x)>0$ for large $|x|$.

The object of this paper is to extend the above results in part to equations (1.1) in which now $f=f(u, x)$ is allowed to depend on $x$. This turns out to be possible if the characteristic scale of the $x$-variable of $f$ is significantly larger than that of the travelling front-like solutions, but not necessarily otherwise. This condition can be expressed by replacing $f(u, x)$ by $f(u, \varepsilon x)$ where $0<\varepsilon \ll 1$. But then we further rescale the space variable by replacing $\varepsilon x$ by $x$ again; this introduces a coefficient $\varepsilon^{2}$ with the second derivative term. We then rescale the time variable to arrive at the equation

$$
\varepsilon u_{t}=\varepsilon^{2} u_{x x}+f(u, x)
$$

This is the equation to be studied here. Throughout, of course, $\varepsilon$ will be assumed to be small "enough" and positive.

The zeros $h_{ \pm}$are now functions of $x$; for convenience we assume the intermediate zero to be at $u=0$ independently of $x$. The more general case when this is not true can be handled with more work along the lines of [1, Section 2]; it cannot however be reduced to the present case by a simple transformation.

The initial value problem for (1.5) with initial data in $S$ and with nonzero derivative at the cross-over point was studied in [1] and the present paper builds upon those results. First, however, they must be strengthened. In [1] it was shown that for small $\varepsilon$, $u(x, t)$ quickly (in time $O(\varepsilon|\ln \varepsilon|)$ develops an internal "layer" of thickness $\leqq O\left(\varepsilon|\ln \varepsilon|^{2}\right)$, which then moves with variable velocity determined by the function $f$. These statements were equipped with estimates which are uniform in bounded time intervals. (Certain uniformity conditions on $f$ were assumed, and we continue to assume them here.) In Section 2 of the present paper, they are shown in fact to be uniform for all time. This improvement, though technical, is highly involved. It is essential for the main result of the paper.

In Section 3, the analogues of $h_{ \pm}$and $u_{ \pm}$for (1.5) are shown to exist. The analogues $H_{ \pm}(x)$ of $h_{ \pm}$are $L_{\infty}$-stable steady solutions; they are no longer constant in $x$. The analogues of $u_{ \pm}$are, as before, connections between $H_{+}$and $H_{-}$, again in the topology of uniform convergence on bounded intervals. Although not strictly travelling waves, these new connections have a front-like spatial structure which moves with variable velocity. They can no longer simply be obtained one from the other by reversal of the sign of $x$; the equation has lost that kind of symmetry. For simplicity we study only $u_{+}$; similar constructions hold for $u_{-}$, of course. Section 3 also contains a good approximate description of the spatial profile of the connection; it remains close to $H_{+}$or to $H_{-}$ except in a layer of width $O(\varepsilon|\ln \varepsilon|)$, and inside that layer it is approximated by the profile of a travelling front in a homogeneous medium. 
It is true here, as before, that a large class of solutions of (1.5) is attracted to a small neighbourhood of the orbit of the connection $u_{+}$or of $u_{-}$. This is shown in Section 4. The size of this neighbourhood is estimated in terms of $\varepsilon$. This is not so strong a result as is known for the homogeneous case, where uniform exponential convergence to a translate of $u_{+}$or $u_{-}$occurs.

A counterexample in Section 5 shows that the assumption that $\varepsilon$ is small is essential for the existence of a connection, all else being the same.

Travelling wave solutions of equations like (1.1) were originally studied by the geneticist R. A. Fisher [6], who used them to model the spread of advantageous genes in a spatially distributed population. In that context, the connections constructed in the present paper can also be interpreted as canonical modes of gene propagation in space when the environment is inhomogeneous; see Section 5 for further discussion.

The formal assumptions on $f$ are as follows; the first two correspond to the first two assumptions in $[1, \S 2]$ :

H1. For each $x, f(u, x)$ has exactly three zeros $h_{-}(x)<0<h_{+}(x)$, these being bounded functions of $x$ which are bounded away from each other, independently of $x$. Also for some $m_{0}>0$,

$$
f_{u}\left(h_{ \pm}(x), x\right)<-m_{0}<0, f_{u}(0, x)>0 ; \quad f(u, x) f_{u u}(u, x) \leqq 0 .
$$

H2. There exist functions $f(u)$ and $f(u)$, each satisfying the analog of H1 (the $h$ 's now being constants and the middle zeros no longer 0 ), such that

$$
\underline{f}(u)<f(u, x)<\bar{f}(u),
$$

the differences between these functions being bounded uniformly away from 0 .

Note that $\mathrm{H} 2$ implies

$$
h_{ \pm}(x) \text { are bounded and bounded away from } 0 .
$$

H3. $f(u, x)$ is smooth, uniformly for $x \in(-\infty, \infty)$ and bounded $u$.

\section{Persistence of layered solutions}

Here, we develop some properties of solutions of the initial value problem for (1.5). They involve a great number of technicalities; the reader who is not interested in these things is invited to read the statements of Theorems 2.1 and 2.10, and turn to Section 3 . First, we recall some preliminary facts and introduce some needed notation.

Because of $\mathrm{H} 1$, it is known that for each fixed $x_{0}$, the equation

$$
u_{t}=u_{x x}+f\left(u, x_{0}\right)
$$

has a travelling wave front solution approaching $h_{ \pm}\left(x_{0}\right)$ as $x \rightarrow \pm \infty$ and moving with a velocity $c\left(x_{0}\right)$. Here $c$ depends smoothly on $x_{0}$ if $f$ does [1] and has sign opposite that 
of $\int_{h_{-}\left(x_{0}\right)}^{h+\left(x_{0}\right)} f\left(u, x_{0}\right) d u$. It is also known that $c$ depends (anti-)monotonically on the function $f\left(u, x_{0}\right)$, and so an easy consequence of $(1.7)$ is that $c(x)$ is bounded independently of $x$ :

$$
|c(x)| \leqq c_{1} .
$$

In [1] it was shown that the trajectories of the internal layers of solutions of (1.5) are given approximately by $x \simeq \psi(t)$, where $\psi$ is a solution of the equation

$$
\psi^{\prime}(t)=c(\psi(t))
$$

This approximation is uniform for $t$ in bounded intervals.

Recall the definition of the set $S$ of functions, given in the preceding section.

Let $u(x, t)$ be the solution of $(1.5)$ satisfying $u(x, 0)=\phi(x)$ for some $\phi \in S$ with

$$
\phi(0)=0 \text { and } \phi^{\prime}(0)>0 .
$$

We assume that $|\phi|$ is small enough that for every $x_{0}, \sup h_{-}<\inf \phi<\sup \phi<\inf h_{+}$, these sup's and inf's being taken over all $x$. This is possible because of (1.8). Then it follows from the maximum principle that

$$
\inf h_{-}(x)<u(x, t)<\sup h_{+}(x) .
$$

for all $x$ and all $t>0$.

It is also a consequence of the maximum principle (see [1] or [3] for example) that $u(\cdot, t) \in S$ for each $t \geqq 0$. Let $Y(t)$ be the unique zero of $u(\cdot, t): u(Y(t), t)=0, Y(0)=0$. For definiteness we define, for each $t_{0}$ and $t>t_{0}$, the function $\psi_{t_{0}}(t)$ to be the solution of (2.2) satisfying $\psi_{t_{0}}\left(t_{0}\right)=Y\left(t_{0}\right)$. The first aim of this section is to prove:

Theorem 2.1. Assume $c(x) \geqq c_{0}>0$ and $\varepsilon$ is small enough. Under Assumptions $H 1$ and $H 2$, there exists a constant $M$ such that for $t>M \varepsilon|\ln \varepsilon|$,

$$
\begin{array}{lll}
\left|u(x, t)-h_{+}(x)\right| \leqq M \varepsilon & \text { for } & x \geqq Y(t)+M \varepsilon|\ln \varepsilon|^{2} \\
\left|u(x, t)-h_{-}(x)\right| \leqq M \varepsilon & \text { for } & x \leqq Y(t)-M \varepsilon|\ln \varepsilon|^{2} .
\end{array}
$$

Also for any $t_{0}$ and $t>t_{0}$,

$$
\left|Y(t)-\psi_{t_{0}}(t)\right| \leqq M\left(\varepsilon|\ln \varepsilon|^{2}+\varepsilon\left(t-t_{0}\right)\right) .
$$

The constant $M$ is independent of $\varepsilon$. Moreover if the initial condition is replaced by $u(x, 0)=\phi\left(x-x_{0}\right)$, then $M$ is also independent of $x_{0}$.

The proof is based on several lemmas whose proofs in turn are based on certain arguments and results in [1]. Generally, we shall use the same notation as in [1], except that the time variable symbol $\tau$ used there is replaced by $t$, and the time variable symbol $t$ used there is replaced by $t / \varepsilon$. 
We define

$$
\tilde{Y}(t) \equiv \operatorname{Max}\{Y(s): 0 \leqq s \leqq t\}
$$

so that $\tilde{Y}$ is piecewise differentiable, $Y(0)=\tilde{Y}(0)$, and

$$
\tilde{Y}^{\prime}(t)>0 \text { implies } \tilde{Y}(t)=Y(t)
$$

The symbols $M, C$ and $m$ in this and later sections refer to various different constants, all of them independent of $\varepsilon$. It will always be assumed, without so stating, that $\varepsilon$ is a small enough positive number.

Lemma 2.2 Given any $\gamma>0$, there are constants $M$ and $m$, independent of $\varepsilon$ but depending on $\gamma$, such that for $t \geqq M \varepsilon|\ln \varepsilon|$ and $x \geqq \tilde{Y}(t)$,

$$
u(x, t) \geqq\left(h_{+}(x)-\gamma\right)\left(1-\exp \left(-m \varepsilon^{-1}(x-\widetilde{Y}(t))\right)\right) .
$$

Proof. First, consider the case when $f$ does not depend on $x$. Then the proof is an adaptation of that of [1, Lemma 3.6]. Define $W(s)$ as in that lemma, and in place of (3.18) there, take

$$
\underline{v}(x, t)=r(t) W(\alpha(t / \varepsilon)(x-\tilde{Y}(t)))
$$

the functions $r$ and $\alpha$ being the same as before. In place of $[1,(3.21)]$, we require

$$
r_{0} W\left(\alpha_{0} x\right) \leqq \phi(x)=u(x, 0) \text { for } x \geqq 0 .
$$

Then as before, $\underline{v}$ will be a lower solution on the domain $\{x>\tilde{Y}(t), t>0\}$ by virtue of the fact that $\tilde{Y}^{\prime}(t) \geqq 0$, and it easily follows, by the exponential convergence of $r$ to $h_{+}$, that

$$
u(x, t) \geqq h_{+}\left(1-e^{-m t}\right)(1-\exp [-m \alpha(t / \varepsilon)(x-\tilde{Y}(t))]) .
$$

For $t \geqq M \varepsilon|\ln \varepsilon|, M$ large enough, we have $\alpha=\varepsilon^{-1}$ and (2.8) follows with $\gamma$ replaced by $\varepsilon$. This implies the conclusion in the case when $f$ does not depend on $x$. The extension to the more general case follows exactly the argument in [1, Theorem 3.10].

Lemma 2.3. For small enough positive $a$ and all $t \geqq 0$, the set $A(a, t) \equiv\{x: u(x, t)<-a\}$ is a nonempty semi-infinite interval $(-\infty, z(a, t))$.

Proof. By (2.3), we know that for $a$ small enough, the equation $\phi(x)=-a$ has a unique solution $x=x(a, 0)$, and $\phi^{\prime}(x)>0$ for $x \in[z(a, 0), 0]$. Thus $A(a, 0)$ is an interval as described, and by continuity so will $A(a, t)$ be for small $t$. If the assertion of the lemma 
were not true, there would be a finite greatest value $t_{1}$ such that $A(a, t)$ is an infinite interval for $0 \leqq t<t_{1}$.

For each $t>0, u(x, t)<-a$ for $x$ in some neighbourhood of $-\infty$. This can be seen, for example, by use of an upper solution

$$
\bar{u}=-(a+\delta)\left(1-\exp \left(x+\varepsilon^{-1} \mu t^{2}+t+1\right)\right)
$$

in the domain $\left\{x<-\varepsilon^{-1} \mu t^{2}-t-1, t>0\right\}$ for sufficiently large positive $\mu$ and small $\delta$ and E. It is known from [1, Theorem 3.2] (note the change in symbol for time) that $u(x, t)<0$ for $x \leqq-M_{0}\left(\varepsilon+\varepsilon^{-1} t^{2}\right), M_{0}$ being the constant in [1, (3.6)]. This inequality for $x$ is satisfied on the right boundary of the comparison domain, for $\mu \geqq M_{0}$. Therefore on the boundary, $\bar{u}=0>u$ and the asserted conclusion follows by the maximum principle.

Therefore $A(a, t)$ must include a neighbourhood of $-\infty$ for every $t$. But it ceases to be connected at $t=t_{1}$, so by smoothness of the function $u$, that function must have an interior maximum of $-a$ at some point $\left(x_{1}, t_{1}\right)$, relative to its values in some cylindrical set $\left\{\left|x-x_{1}\right| \leqq v, t_{1}-\delta \leqq t \leqq t_{1}\right\}$. In fact $u\left(x_{1}, t_{1}\right)=-a>$ the value of $u$ on the lateral and bottom boundaries of the cylinder. In that cylinder, we also have that $h_{-} \leqq u \leqq 0$ by (1.8) for small enough $a$, so

$$
\varepsilon u_{t}-\varepsilon^{2} u_{x x}=f(u \cdot x)<0 .
$$

The interior maximum would contradict the maximum principle, and we conclude that the assertion of the lemma is indeed true.

Lemma 2.4. Let $t_{0}$ satisfy the bound on $t$ given in Lemma 2.2. and suppose that $Y\left(t_{0}\right)=\tilde{Y}\left(t_{0}\right)$. Then for some $m$ independent of $t_{0}$ and $\varepsilon$,

$$
u\left(x, t_{0}\right) \leqq-m\left(1-\exp \left[m \varepsilon^{-1}\left(x-Y\left(t_{0}\right)\right]\right)\right.
$$

for $x \leqq Y\left(t_{0}\right)$.

Proof. Lemma 2.2 with $t=t_{0}$, together with the facts that $Y\left(t_{0}\right)=\tilde{Y}\left(t_{0}\right)$ and $u=0$ when $x=Y\left(t_{0}\right)$, yield

$$
u_{x}\left(Y\left(t_{0}\right), t_{0}\right) \equiv p \geqq m \varepsilon^{-1} .
$$

The bounds in [1, Lemma 3.3] tell us that $\left|u_{x x}\right| \leqq M \varepsilon^{-2}$, so that for $\xi>0$,

$$
u\left(Y\left(t_{0}\right)-\varepsilon \xi, t_{0}\right) \leqq-p \varepsilon \xi+\frac{1}{2} M \xi^{2} \leqq-m \xi+\frac{1}{2} M \xi^{2} .
$$

In particular for $\xi \leqq m / M$, we have that $u \leqq-m_{1} \xi$ for some $m_{1}$. Thus

$$
u \leqq-m \frac{x}{\varepsilon},-m \frac{\varepsilon}{M}<x<0 .
$$


It follows that there is a number $a$, independent of $\varepsilon$, such that $u\left(x_{0}, t_{0}\right)=-a$ for some $x_{0}$ satisfying (2.14). In fact, we can choose $a$ arbitrarily small. It will therefore be assumed to satisfy the hypothesis of Lemma 2.3. Therefore

$$
u\left(x, t_{0}\right) \leqq-a<0 \text { for } x \leqq x_{0}<0 .
$$

This, combined with (2.14), easily yields (2.12) for small enough $m$.

Lemma 2.5. Let $t_{0}=0$ or else $t_{0}$ satisfy the hypothesis of Lemma 2.4. Then there is an $M$ such that

$$
\left|u(x, t)-h_{+}(x)\right| \leqq M \varepsilon
$$

for

$$
\begin{gathered}
x \geqq \psi_{t_{0}}(t)+M\left(\varepsilon|\ln \varepsilon|^{2}+\varepsilon\left(t-t_{0}\right)\right), \\
t \geqq t_{0}+M \varepsilon|\ln \varepsilon| .
\end{gathered}
$$

The inequality (2.16) holds with $h_{+}$replaced by $h_{-}$, if the sense of the inequality in (2.17a) is reversed and the second plus sign on the right is replaced by minus.

Proof. When $t_{0}>0$ satisfies the hypothesis of Lemma 2.2, the Lemmas 2.2 and 2.4 show that the function $u\left(x, t_{0}\right)$ satisfies the hypothesis $\mathrm{H} 3$ on the initial data of the problem considered in [1]. Therefore the conclusions [1, Theorem 3.10 and 4.1] of that paper hold for the present function $u(x, t)$, except with $t$ in those theorems replaced by $t-t_{0}, x$ replaced by $x-Y\left(t_{0}\right)$, and $\psi(t)$ by $\psi_{t_{0}}(t)$. When $t_{0}=0$, this same conclusion is obtained directly. We thus obtain (2.16), (2.17) from [1, Theorem 4.1].

Lemma 2.6. Assume $c(x) \geqq c_{0}>0$ for all $x$. Then under the assumpions of Lemma 2.5, there exists a constant $M_{2}$ and another number $t_{1} \in\left(t_{0}, t_{0}+M_{2} \varepsilon|\ln \varepsilon|^{2}\right)$ such that $Y\left(t_{1}\right)=$ $\tilde{Y}\left(t_{1}\right)$.

Proof. By Lemma 2.5, we know that there exists a constant $M_{1}$ such that $u\left(x, t^{*}\right)<0$ whenever

$$
x<\psi_{t_{0}}\left(t^{*}\right)-M_{1}\left(\varepsilon|\ln \varepsilon|^{2}+\varepsilon\left(t^{*}-t_{0}\right)\right) \text { and } t^{*} \geqq t_{0}+M_{1} \varepsilon|\ln \varepsilon| .
$$

Hence $Y\left(t^{*}\right)>\psi_{t_{0}}\left(t^{*}\right)-M_{1}\left(\varepsilon|\ln \varepsilon|^{2}+\varepsilon\left(t^{*}-t_{0}\right)\right)$, and this in turn is $>Y\left(t_{0}\right)$, provided that

$$
\psi_{t_{0}}\left(t^{*}\right) \geqq Y\left(t_{0}\right)+M_{1}\left(\varepsilon|\ln \varepsilon|^{2}+\varepsilon\left(t^{*}-t_{0}\right)\right) .
$$

But since, by the definition of $\psi_{t_{0}}, \psi_{t_{0}}\left(t^{*}\right)-Y\left(t_{0}\right) \geqq c_{0}\left(t^{*}-t_{0}\right)$, that will be true if we set $t^{*}=t_{0}+\left(2 M_{1} / \mathcal{c}_{0}\right) \varepsilon|\ln \varepsilon|^{2}$ and $\varepsilon$ is of course small enough. So under this condition, 
$\tilde{Y}\left(t^{*}\right) \geqq Y\left(t^{*}\right)>Y\left(t_{0}\right)=\tilde{Y}\left(t_{0}\right)$, and there must be a value $t_{1}$ in the intervening interval $\left(t_{0}, t^{*}\right)$ at which $\tilde{Y}^{\prime}\left(t_{1}\right)>0$, so that $Y=\tilde{Y}$. This completes the proof.

Proof of Theorem 2.1. Lemma 2.6 can be iterated (starting with $t=0$, since $Y(0)=\tilde{Y}(0))$ to produce an increasing sequence $\left\{t_{n}\right\}$ of positive numbers, starting with $t_{0}=0$ and spaced at most a distance $M_{2} \varepsilon|\ln \varepsilon|^{2}$ apart, at which

$$
Y\left(t_{n}\right)=\tilde{Y}\left(t_{n}\right)
$$

If this sequence approaches a finite limit $t_{\infty}$, then by continuity (2.2) holds with $n=\infty$ and we can apply Lemma 2.6 again with $t_{0}=t_{\infty}$, then discard all but a finite number of elements of the sequence near $t_{\infty}$. Hence there is no loss in generality in assuming no finite limit is approached, so $t_{n} \rightarrow \infty$.

By Lemma 2.2 and 2.4, at each $t_{n}$, we have that the function $u\left(x, t_{n}\right)$ is bounded above and below by functions independent of $n$ :

$$
u_{0}(x) \leqq u\left(x-Y\left(t_{n}\right), t_{n}\right) \leqq \tilde{u}_{0}(x),
$$

where these upper and lower bounds each satisfy the hypothesis H3 in [1]. Therefore each function $u\left(x, t_{n}\right)$ may be considered as an initial datum and all the above results hold for the solution it generates, uniformly in $n$.

Therefore by [1, Theorem 4.1], for some $M_{3},(2.5 \mathrm{a})$ holds for

$$
x \geqq \psi_{t_{n}}(t)+M_{3}\left(\varepsilon|\ln \varepsilon|^{2}+\varepsilon\left|t-t_{n}\right|\right), t \geqq t_{n}+M_{3} \varepsilon|\ln \varepsilon| .
$$

But from (2.1), $\psi_{t_{n}}(t) \leqq Y\left(t_{n}\right)+c_{1}\left(t-t_{n}\right)$, so in particular (2.5a) holds for

$$
x \geqq Y\left(t_{n}\right)+2 c_{1}\left(t-t_{n}\right)+M_{3} \varepsilon|\ln \varepsilon|^{2}
$$

and for $t$ in the indicated time interval (2.20). We now restrict $t$ so that

$$
M_{3} \varepsilon|\ln \varepsilon| \leqq t-t_{n} \leqq 2 M_{2} \varepsilon|\ln \varepsilon|^{2} .
$$

This is of course possible if $\varepsilon$ is small enough. Under (2.22), the restriction (2.21) on the values of $x$ may be replaced by

$$
x \geqq Y\left(t_{n}\right)+\left(4 c_{1} M_{2}+M_{3}\right) \varepsilon|\ln \varepsilon|^{2} .
$$

But in the time interval (2.22), we have $\left|Y\left(t_{n}\right)-Y(t)\right|<2 M_{2} c_{1} \varepsilon|\ln \varepsilon|^{2}$, so it suffices to replace $(2.23)$ by

$$
x \geqq Y(t)+\left(6 c_{1} M_{2}+M_{3}\right) \varepsilon|\ln \varepsilon|^{2} .
$$

Setting $M_{4}=6 c_{1} M_{2}+M_{3}$, we conclude that for any given $t \geqq M \varepsilon|\ln \varepsilon|,(2.5)$ holds for 


$$
x \geqq Y(t)+M_{4} \varepsilon|\ln \varepsilon|^{2},
$$

provided only that there is a $t_{n}$ satisfying (2.22) for the given $t$. We show that this is always the case. In fact, let $t_{n}$ be the largest member of the sequence satisfying $t_{n} \leqq t-M_{3} \varepsilon|\ln \varepsilon|$. There is such a member (it might be 0 ) because of the restriction on $t$ given in the statement of the theorem, if the constant $M$ there is $\geqq M_{2}$. This number then satisfies the left side of (2.22), and in fact the right side also because of the maximal spacing of the elements of the sequence. This completes the proof of (2.5a). The analogous proof holds for $(2.5 \mathrm{~b})$; in fact $(2.5 \mathrm{~b})$ holds for

$$
x \geqq \psi_{t_{n}}(t)-M_{3}\left(\varepsilon|\ln \varepsilon|^{2}+\varepsilon\left|t-t_{n}\right|\right),
$$

and the range in $t$ given in (2.16). The rest of the argument is as before.

The estimate (2.6) follows rather directly from [1, Theorem 4.1]. Finally, the last statement of the theorem holds because all the constants involved in the above estimates are independent of where the initial datum changes sign. This completes the proof.

Theorem 2.1 tells us that after an initial transient period, the solution $u$ of the initial value problem remains close to $h_{ \pm}$in regions bounded away from $Y$ by an amount $O\left(\varepsilon|\ln \varepsilon|^{2}\right)$. Our next task is to show a complementary result, namely that in the region close to $Y$, the solution's profile is close to that of a certain travelling wave.

Preliminary to this purpose, it will be convenient to define a refined subset of the set of profiles $S$. Let

$$
\bar{h}_{ \pm} \equiv \sup \underline{h}_{ \pm} ; \underline{h}_{ \pm} \equiv \inf \underline{h}_{ \pm} \text {, }
$$

the sup and inf being taken over $x$. Let $\phi_{*}(\xi)$ and $\phi^{*}(x)$ be bounded monotone increasing functions satisfying, for all $x$,

$$
\begin{gathered}
\phi_{*}(-\infty)<\underline{h}_{-}, 0<\phi_{*}(\infty)<\underline{h}_{+}, \\
0>\phi^{*}(-\infty)>h_{-}, \phi^{*}(\infty)>h_{+}, \phi_{*}(0)<0<\phi^{*}(0) .
\end{gathered}
$$

In addition, the following technical assumption on the functions $\phi_{*}$ and $\phi^{*}$ will be useful. Let $g_{12}(x)$ denote the function appearing on the right of $(2.12)$ and $g_{8}(x)$ the one on the right of (2.8). We require that

$$
g_{12}(x)>\phi_{*}\left(\varepsilon^{-1}\left(x-Y\left(t_{0}\right)\right)\right.
$$

for all $t_{0}$ and all $x<Y\left(t_{0}\right)$, and

$$
g_{8}(x)<\phi^{*}\left(\varepsilon^{-1}(x-\tilde{Y}(t))\right.
$$

for all $t$ and all $x>\widetilde{Y}(t)$, and for some fixed $\delta<h_{+}$independent of $\varepsilon$. These inequalities are easily accommodated by proper choice of $\phi^{*}$ and $\phi_{*}$. 
Finally, let $S_{\varepsilon}^{*}$ be the set of all functions $\psi(x)$ in $S$ such that

$$
\phi_{*}(\xi)<\psi(z+\varepsilon \xi)<\phi^{*}(\xi)
$$

for all $\xi$, where $z$ is the zero of $\psi$.

For any given $t_{0}$ let $x_{0}=Y\left(t_{0}\right), \xi=\left(x-x_{0}\right) / \varepsilon, \tau=\left(t-t_{0}\right) / \varepsilon, c_{(0)}=c\left(x_{0}\right)$, and $v(\xi, \tau)$ the solution of

$$
\begin{gathered}
v_{\tau}=v_{\xi \xi}+f\left(v, x_{0}\right), \tau>0, \\
v(\xi, 0)=u\left(x_{0}+\varepsilon \xi, t_{0}\right) .
\end{gathered}
$$

Let $V\left(\xi-c_{(0)} \tau\right)$ be the travelling front solution of (2.26a) approaching $h_{ \pm}\left(x_{0}\right)$ as $\xi \rightarrow \pm \infty$, normalized so that $V(0)=0$. This normalization will always be used when we speak of travelling fronts.

By [2], we know that if $u\left(\cdot, t_{0}\right) \in S_{\varepsilon}^{*}$ then for some $\xi_{0}, M_{2}$ and $\alpha$, the function $v$ satisfies

$$
\left|v(\xi, \tau)-V\left(\xi-c_{(0)} \tau-\xi_{0}\right)\right| \leqq M_{2} e^{-\alpha \tau}
$$

In [2, Theorem 3.1], this was proved for $M_{2}$ and $\alpha$ possibly depending on $x_{0}$ and the initial datum $u\left(\cdot, t_{0}\right)$. We shall need the estimate, however, with uniform $M_{2}$ and $\alpha$.

Lemma 2.7. The constants $M_{2}$ and $\alpha$ in (2.27) are independent of $t_{0}$ (hence $x_{0}$ ), $\varepsilon$, and $u\left(\cdot, t_{0}\right)$, as long as the latter is in $S_{\varepsilon}^{*}$.

The proof is a refinement of the proof of [2, Theorem 3.1.] and is given in the appendix.

Now let $w(\xi, \tau) \equiv u\left(x_{0}+\varepsilon \xi, t_{0}+\varepsilon \tau\right)-v(\xi, \tau)$, so that

$$
\begin{gathered}
w_{\tau}-w_{\xi \xi}=f\left(u, x_{0}+\varepsilon \xi\right)-f\left(v, x_{0}\right)=a(\xi, \tau) w+g(\xi, \tau), \\
w(\xi, 0)=0,
\end{gathered}
$$

where

$$
\left.a(\xi, \tau) \equiv f_{u}(v(\xi, \tau)+\theta w(\xi, \tau)), x_{0}\right), g(\xi, \tau) \equiv f\left(u, x_{0}+\varepsilon \xi\right)-f\left(u, x_{0}\right),
$$

$0<\theta<1$. Because of the smoothness and boundedness of $f, g$ can clearly be estimated as follows. Let $\Psi(\xi)$ be a function such that for some $C$,

$$
|\xi|<\Psi(\xi)<1+|\xi| ; \Psi^{\prime \prime}(\xi) \leqq C \Psi(\xi) .
$$

Then for some $C$,

$$
|g(\xi, \tau)| \leqq C \varepsilon \Psi(\xi)
$$


Lemma 2.8. For some $M_{1}$ and $\mu$ independent of $\varepsilon$ and $x_{0}$,

$$
|w(\xi, \tau)| \leqq M_{1} \varepsilon(|\xi|+1) e^{\mu \tau}
$$

Proof. Let $\bar{w}(\xi, \tau) \equiv M_{1} \varepsilon \Psi(\xi) e^{\mu \tau}$. Then by (2.30) and (2.31),

$$
\left(\partial_{\tau}-\partial_{\xi \xi}-a(\xi, \tau)\right) \bar{w}=\mu \bar{w}-M_{1} \varepsilon \Psi^{\prime \prime}(\xi) e^{\mu \tau}-a \bar{w} \geqq(\mu-C-a) \bar{w} \geqq \frac{1}{2} M_{1} \mu \varepsilon \Psi(\xi) \geqq g(\xi, \tau)
$$

for $\mu \geqq 2(C+a(\xi, \tau))$ and $\mu \geqq 2 C / M_{1}$. Therefore $\bar{w}$ is an upper solution for (2.28), and we have $w \leqq \bar{w}$, which provides (2.32) with the absolute value signs omitted on the left. A similar lower solution will complete the proof.

Lemma 2.29. Let $u\left(\cdot, t_{0}\right) \in S_{\varepsilon}^{*}$. For some $\xi_{0}$ there are constants $\sigma, v, C$, and $m_{2}$, independent of $\varepsilon$, with $0<\sigma, v<1$, such that

$$
\left|u\left(x_{0}+\varepsilon \xi, t_{0}+\varepsilon \tau\right)-V\left(\xi-c_{0} \tau-\xi_{0}\right)\right| \leqq \varepsilon^{v}
$$

for

$$
C|\ln \varepsilon| \leqq \tau \leqq 2 C|\ln \varepsilon|,|\xi| \leqq m_{2} \varepsilon^{-\sigma} .
$$

Proof. For any $\delta>0$, we have by (2.32) that $|w|<\delta$ for $\varepsilon(|\xi|+1)<1$ and

$$
\tau \leqq-\frac{1}{\mu} \ln M_{1}+\frac{1}{\mu}(|\ln \varepsilon| \xi||-|\ln \delta|),
$$

and if we assume that

$$
|\ln \delta|<\frac{1}{2}|\ln \varepsilon| \xi||
$$

then this is true for

$$
\tau \leqq-M_{3}+(2 \mu)^{-1}|\ln \varepsilon \xi|
$$

$M_{3}$ depending on $\mu$ and $M_{1}$.

We also have from (2.27) that $|v-V|<\delta$ for

$$
\tau \geqq \alpha^{-1}\left|\ln \frac{\delta}{M_{2}}\right| .
$$

We shall require that

$$
\alpha^{-1}\left|\ln \frac{\delta}{M_{2}}\right|<\frac{1}{4}\left(-M_{3}+(2 \mu)^{-1} \mid \ln \varepsilon \xi\right)
$$


and

$$
\delta=\frac{1}{2} \varepsilon^{v}
$$

for some $v>0$. Therefore since $|u-V| \leqq|u-v|+|w|$, (2.33) will hold for

$$
\alpha^{-1}\left|\ln \frac{\delta}{M_{2}}\right|<\tau<4 \alpha^{-1}\left|\ln \frac{\delta}{M_{2}}\right| \text {. }
$$

But (2.38) and (2.39) are equivalent to

$$
|\xi|<m_{2} \varepsilon^{-\sigma}
$$

where

$$
\sigma=1-8 \mu \alpha^{-1} v
$$

and $m_{2}$ depends on the other constants. This is the requirement on the right of (2.34).

We now choose $v$ so that

$$
0<v<\frac{\alpha}{8 \mu},
$$

so $0<\sigma<1$. Then (2.35) can be checked and found to follow from (2.41) for small enough, $\varepsilon$, provided $4 \mu \alpha^{-1}>1$, which can be arranged by increasing the size of $\mu$, if necessary.

We also restrict $\varepsilon$ to be so small that

$$
v^{-1} \ln \left(M_{2} / 2\right)<|\ln \varepsilon|
$$

and let $C=\alpha^{-1} v$ in (2.34). By simple algebra, the left part of (2.34) now implies (2.40), which in turn implies (2.33), completing the proof.

In the following, we use the symbol $V_{(t)}(z)$, for $t$ fixed and $z=\xi-c(Y(t)) \tau$, to denote the travelling front solution of (2.26a) with the constant $x_{0}$ replaced by $Y(t)$, and normalized to vanish when $z=0$.

Theorem 2.10. Let $c(x) \geqq c_{0}>0$. Then for all $t \geqq M \varepsilon|\ln \varepsilon|^{2}$, we have (2.5), and for some $\xi_{0}(t)$

$$
\left|u(Y(t)+\varepsilon \xi, t)-V_{(t)}\left(\xi-\xi_{0}(t)\right)\right|<\varepsilon^{v}
$$

for $|\xi|<m_{2} \varepsilon^{-\sigma}$, where $m_{2}, v$, and $\sigma$ are the same as in Lemma 2.9.

Note. This theorem shows that near the position $x_{0}$, where the function $u(\cdot, t)$ 
vanishes, it has a profile which can be approximated by the travelling front profile of (2.26a) associated with that value of $x_{0}$.

Proof. First, we show that $u(\cdot, t) \in S_{\varepsilon}^{*}$ for all $t$ subject to the stated restriction.

Let $t_{0}$ be such that $u\left(\cdot, t_{0}\right) \in S_{\varepsilon}^{*}$. Let $\tau$ satisfy the left part of (2.34), but

$$
\xi>m_{2} \varepsilon^{-\sigma} .
$$

Then using the constants $M, C$, and $c_{1}$ from (2.5), (2.34), and (2.1), and setting $x=x_{0}+\varepsilon \xi, t=t_{0}+\varepsilon \tau$, we have

$$
\begin{aligned}
x & =x_{0}+\varepsilon \xi=Y\left(t_{0}\right)+\varepsilon \xi>Y(t)+Y\left(t_{0}\right)-Y\left(t_{0}+\varepsilon \tau\right)+m_{2} \varepsilon^{1-\sigma} \\
\geqq & Y(t)-c_{1} \varepsilon \tau+m_{2} \varepsilon^{1-\sigma} \\
& >Y(t)-2 c_{1} C \varepsilon|\ln \varepsilon|+m_{2} \varepsilon^{1-\sigma} \\
> & Y(t)+M \varepsilon|\ln \varepsilon|^{2}
\end{aligned}
$$

for $\varepsilon$ small enough. Therefore by Theorem 2.1, (2.5a) holds. Similarly, (2.5b) holds for $\xi<-m_{2} \varepsilon^{-\sigma}$.

This means that for $|\xi|>m_{2} \varepsilon^{-o}$ and the left part of (2.34) satisfied, $u(x, t)$ differs from $h_{+}(x)$ by an amount $\leqq O(\varepsilon)$. On the other hand for $|\xi| \leqq m_{2} \varepsilon^{-\sigma}$, Lemma 2.9 shows that $u$ differs from $V$ by an amount $\leqq \varepsilon^{v}$. Combining the two clearly implies that $u(\cdot, t) \in S_{\varepsilon}^{*}$ for $C|\ln \varepsilon| \leqq \tau \leqq 2 C|\ln \varepsilon|$.

We apply Lemma 2.9 again, replacing $t_{0}$ by $t_{0}+\varepsilon \tau$ for all possible $\tau$ in that allowed range, and so obtain that $u(\cdot, t) \in \mathrm{S}_{\varepsilon}^{*}$ for $C|\ln \varepsilon| \leqq \tau \leqq 4 C|\ln \varepsilon|$. Continuing this iteration procedure, we find that $u(\cdot, t) \in S_{\varepsilon}^{*}$ for all $\tau \geqq C|\ln \varepsilon|$, i.e.

$$
t \geqq t_{0}+\varepsilon C|\ln \varepsilon|,
$$

subject only to the requirement that $u\left(\cdot, t_{0}\right) \in S_{\varepsilon}^{*}$. This latter would follow in turn from (2.12), (2.8), and the technical restrictions (2.25), if $Y\left(t_{0}\right)=\tilde{Y}\left(t_{0}\right)$ and $t_{0} \geqq M \varepsilon|\ln \varepsilon|$. However by Lemma 2.6 with $t_{0}$ set equal to 0 in that lemma, we know that these conditions do hold for some point $t_{1} \in\left(0, M \varepsilon|\ln \varepsilon|^{2}\right)$. Take that point to be $t_{0}$; we use (2.44) to obtain that $u(\cdot, t) \in S_{\varepsilon}^{*}$ for $t$ in the range

$$
t \geqq M \varepsilon|\ln \varepsilon|^{2} .
$$

For each fixed $t$ in this range, we may now apply Lemma 2.9, setting $x_{0}=Y(t)$, $\tau=C|\ln \varepsilon|$ fixed, and replacing the expression $-c_{(0)} \tau-\xi_{0}$ in (2.33) by $-\xi_{0}$; it clearly depends on $t$. We then obtain (2.43) with the second argument " $t$ " of $u$ replaced by $t+C \varepsilon|\ln \varepsilon|$. Now (2.43) as written can be obtained by observing that $u(\cdot, t+C \varepsilon|\ln \varepsilon|)$ differs from $u(\cdot, t)$ by a quantity uniformly small of order $C_{\varepsilon}|\ln \varepsilon|$; one need only reduce 
the exponent $v$ by a small amount and require $\varepsilon$ to be small enough. This completes the proof.

\section{Existence of a connection}

Theorem 3.1. For small enough $\varepsilon$, there exist stationary solutions $H_{ \pm}(x)$ of (1.5) satisfying

$$
\left|H_{ \pm}(x)-h_{ \pm}(x)\right|<M \varepsilon
$$

for some $M$ independent of $\varepsilon$. Moreover for any fixed $M$, either choice of the subscript, and small enough $\varepsilon$, there is at most one stationary solution satisfying (3.1). They are exponentially stable in the $L_{\infty}$ norm.

Proof. Define the operator $\mathcal{N}$ by

$$
\mathcal{N} u \equiv \varepsilon u_{t}-\varepsilon^{2} u_{x x}-f(u, x) .
$$

A $t$-independent upper solution in the required neighbourhood of $h_{+}$can be constructed by setting

$$
\vec{u}(x) \equiv h_{+}(x)+\mu \varepsilon,
$$

for $\mu$ sufficiently large; in fact since by $(1.6) f_{u}(u, x) \leqq-m<0$ for $u$ in a neighbourhood of $h_{ \pm}(x)$, we have

$$
\mathscr{N} \bar{u}=-\varepsilon^{2} \bar{u}_{x x}-f\left(h_{+}(x)+\mu \varepsilon, x\right) \geqq-\varepsilon^{2} h_{-}^{\prime \prime}(x)+m \mu \varepsilon \geqq 0
$$

for large $\mu$ independent of $\varepsilon$. A corresponding lower solution is obtained by replacing $\mu$ by $-\mu$. Since the lower solution is below the upper one, there exists an exact stationary solution $H_{+}(x)$ between them, and it satisfies (3.1) with $M=\mu$. The existence of $H_{-}$is obtained the same way.

Now suppose there were two solutions $H_{+}^{(1)}$ and $H_{+}^{(2)}$ satisfying (3.1) for some fixed $M$. The difference $w(x)$ of the two satisfies a linear differential equation of the form $\varepsilon^{2} w^{\prime \prime}-a(x) w=0$, with $a(x)>0$ for small enough $\varepsilon$. The maximum principle shows that $w \equiv 0$, establishing uniqueness.

Finally, the fact that $f_{u}<0$ in a neighbourhood of $H_{ \pm}$shows that there is a lower solution of the form $H_{+}(x)-\delta e^{-m t}$ for small enough $\delta$ and $m$. This and the upper solution obtained by replacing $\delta$ by $-\delta$ shows $H_{+}$to be exponentially stable in $L_{\infty}$.

Lemma 3.2. There exists an $m>0$ such that if, for some $x_{0}, t_{0}$, and $T$ and for some small enough $\delta$ (independent of $\varepsilon$ ),

$$
\left|u(x, t)-H_{+}(x)\right|<\delta
$$


for $x>x_{0}, t_{0}<t<T$, then

$$
\left|u(x, t)-H_{+}(x)\right| \leqq \delta\left(e^{-m\left(x-x_{0}\right)}+e^{-m\left(t-t_{0}\right)}\right)
$$

for $x>x_{0}, t_{0}<t<T$. Moreover if

$$
\left|u(x, t)-H_{-}(x)\right|<\delta
$$

for $x<x_{0}, t_{0}<t<T$, then

$$
\left|u(x, t)-H_{-}(x)\right| \leqq \delta\left(e^{-m\left(x_{0}-x\right)}+e^{-m\left(t-t_{0}\right)}\right)
$$

for $x<x_{0}, t_{0}<t<T$.

Proof. Let $w(x, t) \equiv \delta\left(e^{-\alpha x}+e^{-\alpha t}\right)$ and $\bar{v}(x, t) \equiv H_{+}(x)+w\left(x-x_{0}, t-t_{0}\right)$. Then since $H_{+}$ is an exact solution,

$$
\mathcal{N} \bar{v}(x, t)=-\left(\varepsilon \alpha+\varepsilon^{2} \alpha^{2}\right) w-\varepsilon^{2} H_{+}^{\prime \prime}(x)-f\left(H_{+}+w, x\right)=-\left(\varepsilon \alpha+\varepsilon^{2} \alpha^{2}\right) w-f_{u}\left(H_{+}+\theta w\right) w,
$$

where $0<\theta<1$. Now since $f_{u}\left(H_{+}+\theta w\right)<0$ for $\delta$ and $\varepsilon$ sufficiently small, we have $\mathcal{N} \bar{v} \geqq 0$ for small enough $\varepsilon \alpha$. Moreover assuming (3.3), we have $\bar{v} \geqq u$ for $x=x_{0}, t \in\left(t_{0}, T\right)$, and also for $t=t_{0}, x \geqq x_{0}$. Therefore $\bar{v}$ is an upper solution for (1) in the domain $x>x_{0}$, $t_{0}<t<T$, and we conclude that in this domain

$$
u(x, t) \leqq \bar{v}(x, t) .
$$

A corresponding lower estimate can be derived, and together they give (3.4) with $m=\alpha$. The other assertion of the lemma is established the same way.

For the following, we recall the velocity function $c(x)$ used in (2.1).

Theorem 3.3. Assume

$$
c(x)>c_{0}>0 \text { for all } x .
$$

If $\varepsilon$ is small enough, there exists a solution $u_{\infty}(x, t)$ of $(1.5)$ for all real $x$ and $t$, satisfying

$$
\lim _{x \rightarrow \pm \infty}\left|u_{\infty}(x, t)-H_{ \pm}(x)\right|=0 \text { for all } t
$$

$\lim _{t \rightarrow \mp \infty} u_{\infty}(x, t)=H_{ \pm}(x)$ uniformly for $x$ in bounded intervals;

$u_{\infty}$ has a unique zero $Y_{\infty}(t)$ for each $t$ satisfying 


$$
Y_{\infty}( \pm \infty)= \pm \infty
$$

and $u_{\infty}$ satisfies the estimates given in Theorem 2.10 for all $t$.

Remark. This is our most basic result. It establishes the existence of a connection from $H_{+}$to $H_{-}$, and also shows that the connection is in the form of a solution with a single moving internal layer. The approximate law of motion of this layer is given by (2.2) and (2.6). Finally, it characterizes the inner structure of the layer as being near that of a wave front for the homogeneous equation, the argument $x$ in (1.5) being frozen.

Proof. For each integer $n>0$, let $u_{n}(x, t)$ be the solution of (1.5) with initial datum at some $t=t_{n}$ to be given below: $u_{n}\left(x, t_{n}\right)=\phi^{*}(x+n)$. Let $Y_{n}(t)$ denote the position of the unique zero of $u_{n}(x, t)$. By Theorems 2.1 and 2.10, and (3.7), we know that $u_{n}$ develops a layer which propagates indefinitely far to the right. (Note that $\psi_{t_{0}}(t) \geqq Y\left(t_{0}\right)+c_{0}\left(t-t_{0}\right)$.) This implies that

$$
\lim _{t \rightarrow \infty} Y_{n}(t)=\infty .
$$

We define the numbers $t_{n}<0$, used above, so that

$$
Y_{n}(0)=0 \text { for all } n \text {. }
$$

This is clearly possible because of (3.12) and the fact that $Y_{n}\left(t_{n}\right)=-n$. Moreover the bound on speed of propagation implied by (2.1) together with Theorem 2.1 shows that

$$
t_{n} \rightarrow-\infty \text { as } n \rightarrow \infty \text {. }
$$

By (3.14), for each $K$ there exists an $N(K)$ such that the set of functions $\left\{u_{n}\right\}$ for $n>N$, together with their derivatives up to some order, are equicontinuous in the region

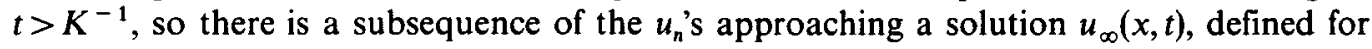
all $t$ as well as for all $x$. The approach is uniform on bounded sets in the $(x, t)$ plane.

Each $u_{n}$ satisfies the estimates in Theorem 2.10 for $t \geqq t_{n}+M \varepsilon|\ln \varepsilon|^{2}$, so the limit $u_{\infty}$ does also, for each $t$ without restriction.

Those estimates imply, of course, that $u_{\infty}(\cdot, t)$ has at least one $0, Y_{\infty}(t)$.

To show that $Y_{\infty}$ is unique, we argue as follows. Given any interval $I$ on the $x$-axis of length $M_{2} \varepsilon|\ln \varepsilon|^{2}\left(M_{2}\right.$ as in Lemma 2.6), there exists a sequence of values $\tau_{n}, n=n^{*}$, $n^{*}+1, \ldots$, with $n^{*}$ sufficiently large, such that $Y_{n}\left(\tau_{n}\right)=\tilde{Y}_{n}\left(\tau_{n}\right) \in I$ for each $n$ in the sequence. This follows by iterating Lemma 2.6 as in the proof of Theorem 2.1. We have from Lemma 2.2 that

$$
\partial_{x} u_{n}\left(Y_{n}\left(\tau_{n}\right), \tau_{n}\right) \text { is bounded away from } 0 \text {, independently of } n .
$$

By the construction of the functions $u_{n}$, the sequence $\left\{\tau_{n}\right\}$ will be bounded. Take a subsequence along which $\tau_{n} \rightarrow \tau_{\infty}, Y_{n}\left(\tau_{n}\right) \rightarrow Y_{\infty} \in I$, and $u_{n}(x, t) \rightarrow u_{\infty}(x, t)$ for $x \in I$ and $t$ in a 
neighbourhood of $\tau_{\infty}$. Then $u_{\infty}\left(Y_{\infty}, \tau_{\infty}\right)=0$ and by (3.17), $\partial_{x} u_{\infty}\left(Y_{\infty}, \tau_{\infty}\right)>0$. The function $u_{\infty}\left(\cdot, \tau_{\infty}\right)$ cannot vanish at another place besides $x=Y_{\infty}$, for then by convergence to $u_{\infty}$, there would be a function $u_{n}$ which takes on arbitrarily small values (for large enough $n$ ) at a point bounded away from $Y_{n}\left(\tau_{n}\right)$. If these values are negative, this would contradict Lemma 2.3 and (3.17). But the analogue of Lemma 2.3 also holds for positive $u$. This shows that $Y_{\infty}$ is unique.

Now (3.10) with the + sign follows from (3.12), and the - sign follows from (3.14).

It only remains to establish (3.8) and (3.9). Given numbers $\delta>0$ and $x_{0}$, let $T\left(x_{0}, \delta\right)$ be the largest time such that $\left|u_{\infty}(x, t)-H_{+}(x)\right|<\delta$ for $x \geqq x_{0}, t \leqq T\left(x_{0}, \delta\right)$. Then from (3.10) and (3.11)

$$
T\left(x_{0}, \delta\right) \rightarrow \pm \infty \text { as } x_{0} \rightarrow \pm \infty .
$$

Now fix $t$ and let $x_{0}$ be such that $T\left(x_{0}, \delta\right)>t$ (this is possible by (3.18)). Use these values of $x_{0}$ and $T$ in (3.4). Let $x \rightarrow \infty$ and $t_{0} \rightarrow-\infty$ in that inequality to obtain (3.8) with the plus sign.

Similarly, let $T^{*}\left(x_{0}, \delta\right)$ be the smallest time such that $\left|u_{\infty}(x, t)-H_{-}(x)\right|<\delta$ for $x \leqq x_{0}$, $t \geqq T^{*}\left(x_{0}, \delta\right)$ with $x_{0}$ restricted to be negative and so large that $t_{0}<t$. In (3.6), first let $x \rightarrow-\infty$; then let $x_{0}$ and hence $t_{0}$ approach $-\infty$. This yields (3.8) with the minus sign.

On the other hand in (3.6) if we fix $x$, let $t \rightarrow \infty$, and then let $x_{0}$ and $t_{0}=T^{*}\left(x_{0}, \delta\right)$ approach $+\infty$, we obtain (3.9) with $H_{-}$. A similar argument will give (3.9) with $H_{+}$. This completes the proof.

\section{Attraction to the connection}

The following theorem shows that initial data in $S$ are rapidly (in time $O\left(\varepsilon|\ln \varepsilon|^{2}\right.$ ) attracted to a neighbourhood of the orbit of $u_{\infty}$. This neighbourhood is uniformly of size $O\left(\varepsilon^{v}\right)$ for some $v>0$. At locations at least a distance $O\left(\varepsilon|\ln \varepsilon|^{2}\right)$ away from the layer, the closeness estimate is improved to $O(\varepsilon)$.

Theorem 4.1. Let $u(x, t)$ be a solution of $(1.5)$ with $u(\cdot, 0) \in S$, under the assumptions of Theorem 2.1. There are positive constants $M$ and $v$, and a function $\bar{\psi}(t)$ such that

$$
\left|u(x, t)-u_{\infty}(x, \bar{\psi}(t))\right|<M \varepsilon^{\nu}
$$

for $t \geqq M \varepsilon|\ln \varepsilon|^{2}$. Moreover,

$$
\left|u(x, t)-u_{\infty}(x, \bar{\psi}(t))\right|<M \varepsilon
$$

for $t \geqq M \varepsilon|\ln \varepsilon|^{2},|x-Y(t)|>M \varepsilon|\ln \varepsilon|^{2}$.

Here for each $t_{0}$ and $t>t_{0}$,

$$
\left|\psi(t)-\psi_{t_{0}}(t)\right|<M \varepsilon\left(t-t_{0}\right)
$$

Proof. Choose $t_{0}$ so that $Y_{\infty}\left(t_{0}\right)=Y(0)$. 
Then $u(x, t)$ and $u_{\infty}\left(x, t-t_{0}\right)$ will both satisfy the estimates of Theorem 2.10 for $t \geqq M \varepsilon|\ln \varepsilon|^{2}$. Hence (4.1) follows from the fact that

$$
\left|u-u_{\infty}\right| \leqq|u-V|+\left|u_{\infty}-V\right|
$$

and similarly for (4.2).

\section{The question of connections and barriers when $\varepsilon$ is not small}

The assumption in Theorem 3.3 that $\varepsilon$ is small enough was crucial to the argument. Suppose that $\varepsilon=1$, for example. Then conditions were given in [4] for there to exist a stationary solution $u_{0}(x)$ of (1.5) which satifies (3.8) with $u_{\infty}$ replaced by $u_{0}$ (but not (3.9), of course, since $u_{0}$ is independent of $t$ ). Stationary solutions of this type were called clines there because of their relevance to geographic problems in population genetics. It can be shown [5] that in some sense clines, if they exist, block the propagation of wave fronts whose profiles are monotone in the same direction as that of the cline.

Here we merely give an example to illustrate this blockage phenomenon. Specifically, we show that a certain sufficient condition given in [4] for the existence of a cline will also prevent the existence of a connection $u_{\infty}$, in the sense of this paper.

The case in point is when $f(u, x)=s(x) f(u)$, where $s(x)$ is identically one for $|x| \geqq b$ for some $b$, and for $|x| \leqq b$,

$$
s(x)=(1+\mu \theta(x, b))^{-1}, \mu>0 .
$$

Here the function $\theta$ is smooth and nonnegative, vanishes for $|x|>b$, and has a maximum of 1 . In [4, Theorem 4], it was shown that if

$$
8 b^{2} \int_{0}^{h_{+}} f(r) d r>h_{-}^{2}
$$

then a cline exists for sufficiently large $\mu$. In fact, a stationary lower solution $\underline{u}(x)$ was constructed there, of the form (for some $p$ with $|p|<b$ )

$$
\underline{u}(x)=h_{-}, x<p, \quad \underline{u}(x)=v_{1}(x), p \leqq x \leqq b, \quad \underline{u}(x)=v_{2}(x), p>b,
$$

where $v_{1}(p)=0, v_{1}(b)=0=v_{2}(b), v_{2}(\infty)=h_{+}$, and $v_{1}$ and $v_{2}$ satisfy certain autonomous differential equations.

It can be checked that under $H 1\left(f^{\prime \prime}(u) \leqq 0\right.$ for $\left.u>0\right)$, another lower solution $\underline{u}$ can also be constructed with

$$
\underline{u}(\infty)=\gamma h_{+},
$$


where $\gamma<1$ and $1-\gamma$ is sufficiently small. Specifically, one replaces $v_{2}(x)$ above by $\gamma v_{2}(x)$. Then if $u^{-1} f(u)$ is a monotone decreasing function of $u$ for $u>0$ (which it will be if $f^{\prime \prime}(u)<0$ ), then $\gamma v_{2}$ is a lower solution. Then $v_{1}$ and $p$ can be adjusted in an obvious manner.

Note that the new $\underline{u}$ is bounded above by $\gamma h_{+}<h_{+}$, and is identically $h_{-}$for $x<-b$. If a connection $u_{\infty}(x, t)$ existed, it would satisfy $h_{-}<u_{\infty}<h_{+}$, so for sufficiently large negative $t$ would lie above $\underline{u}$. By the maximum principle, it would have to lie above $\underline{u}$ for all $t$, and so could never attain a neighbourhood of the stationary state $h_{-}$. So it could not be a connection.

It is natural to expect that if a cline $u_{0}(x)$ exists and no other stationary solution lies between it and $h^{+}$, then a connection will exist from $h_{+}$to $u_{0}$. Under certain conditions, this can be shown to be the case; we shall not pursue that point here.

\section{Appendix: Proof of Lemma 2.7}

Denote $z=\xi-c_{(0)} \tau$ and $\tilde{v}(z, \tau)=v\left(z+c_{(0)} \tau, \tau\right)$. We shall work with $\tilde{v}$ instead of $v$; however to simplify the notation, we drop the "tilde" and write $v(z, \tau)$. The constants $v$ and $\mu$ below are not the same as in Section 2 .

Let $\phi(\xi)=u\left(x_{0}+\varepsilon \xi, t_{0}\right)$. From (2.26), then, $v(z, \tau)$ will be the solution of

$$
\begin{gathered}
v_{\mathrm{r}}-c_{(0)} v_{z}-v_{z z}-f\left(v, x_{0}\right)=0, \tau>0, \\
v(z, 0)=\phi(z) .
\end{gathered}
$$

The assumption was that

$$
\phi(z) \in\left(\phi_{*}(z), \phi^{*}(z)\right)
$$

The proof of Lemma 2.7 depends on certain uniform continuity results, which we now develop. Let $f(v, \lambda)$ be a smooth function which is bistable (i.e., H1 holds) for each $\lambda$ in some bounded open interval $\Lambda$ on the real line. Also, let $\phi(z ; \lambda)$ be a smooth function for all real $z$ and $\lambda$ in $\Lambda$ which, as a function of $z$, is in $S$ for each $\lambda$. It is continuous in the uniform norm on the real line, which we denote by $|\cdot|_{0}$. Let $c(\lambda)$ be the speed and $V(z ; \lambda)$ the profile of the travelling front solution with nonlinearity $f(v, p)$. Thus

$$
c(\lambda) V_{z}+V_{z z}+f(V, \lambda)=0, V( \pm \infty ; \lambda)=h_{ \pm}(\lambda), V(0 ; \lambda)=0 .
$$

Let $v(z, \tau, \lambda)$ be the solution of the initial value problem (A.1) with $c_{(0)}$ replaced by $c(\lambda)$, $f\left(v, x_{0}\right)$ by $f(v, \lambda)$, and $\phi(z)$ by $\phi(z ; \lambda)$.

By [2], we know there exists a number $z_{0}(\lambda)$ such that

$$
\left|v(z, \tau ; \lambda)-V\left(z-z_{0}(\lambda) ; \lambda\right)\right|_{0} \rightarrow 0 \text { as } \tau \rightarrow \infty .
$$

Let the function $\alpha(\tau ; \lambda)$ be such that 


$$
\int_{-\infty}^{\infty} e^{c(\lambda) z} h(z, \tau ; \lambda) V^{\prime}\left(z-z_{0}(\lambda)-\alpha(\tau ; \lambda) ; \lambda\right) d z=0
$$

where

$$
h(z, \tau ; \lambda)=v(z, \tau ; \lambda)-V\left(z-z_{0}(\lambda)-\alpha(\tau ; \lambda) ; \lambda\right)
$$

so that

$$
\alpha(\infty, \lambda) \equiv 0
$$

It was shown in $[2, \S 5]$ that for each $\lambda$ a unique function $\alpha(\tau ; \lambda)$, continuous in $\tau$ and satisfying (A.5), (A.7), exists for $\tau$ sufficiently large, say $\tau>\tau^{*}(\lambda)$.

The functions $h$ and $\alpha$ figure into the proof of exponential convergence in [2].

\section{Lemma A.1.}

(i) The functions $c(\lambda)$ and $z_{0}(\lambda)$ are continuous;

(ii) $V(z ; \lambda)$ is continuous in $\lambda$, uniformly in $z$;

(iii) $\alpha(\tau ; \lambda)$ is continuous in $\lambda$, uniformly for $(\tau, \lambda)$ in a neighbourhood of $(\infty, 0)$. Also $\tau^{*}(\lambda)$ is bounded for $\lambda$ in a neighbourhood of 0 ;

(iv) $v(z, \tau ; \lambda)$ and hence $h(z, \tau ; \lambda)$ are continuous, uniformly for all $z$ and for bounded $\tau>\tau^{*}(\lambda) ;$ and

(v) for any $\delta>0$, there exist numbers $T$ and $\varepsilon_{1}$ such that $|h|_{0}<\delta$ for $\tau=T$ and $|\lambda|<\varepsilon_{1}$.

Proof. The continuity of $c$ and $V$ were proved in [1, Appendix]. Consider $z_{0}(\lambda)$. It suffices to prove its continuity at the point $\lambda=0$, which we suppose lies in $\Lambda$. Given any $\delta>0$, let $T=T(\delta)$ be such that

$$
\left|v(z, \tau ; 0)-V\left(z-z_{0}(0), 0\right)\right|_{0}<\delta
$$

for $\tau>T$ (possible by (A.4)).

Next, let $\varepsilon_{1}$ be such that

$$
|V(z, 0)-V(z, \lambda)|_{0}<\delta
$$

and

$$
|v(z, T ; 0)-v(z, T ; \lambda)|_{0}<\delta
$$

for $|\lambda|<\varepsilon_{1}$. The first of these two requirements is possible by (ii); the latter is possible by the continuity of $v$ with respect to $\lambda$ in the uniform norm, uniformly for bounded $\tau$ intervals. This in turn is a standard result which can be proved with the maximum principle. (Incidentally, it establishes the continuity of $v$ in (iv).)

For this range of $\lambda$, these last three inequalities may be added to yield 


$$
\left|v(z, T ; \lambda)-V\left(z-z_{0}(0), \lambda\right)\right|_{0} \leqq 3 \delta .
$$

By [2, Lemma 4.2], it follows that $\left|v(z, \tau ; \lambda)-V\left(z-z_{0}(0), \lambda\right)\right|_{0}$ can be made to stay arbitrarily small for all $\tau \geqq T(\delta)$ by choosing $\delta$, hence $\varepsilon_{1}$, to be sufficiently small. But its limit as $\tau \rightarrow \infty$ is $\left|V\left(z-z_{0}(\lambda), \lambda\right)-V\left(z-z_{0}(0), \lambda\right)\right|_{0}$, which is therefore arbitrarily small when $\lambda$ is sufficiently small. But this norm is $\geqq m\left|z_{0}(\lambda)-z_{0}(0)\right|$ for some $m>0$, so $z_{0}$ depends continuously on $\lambda$.

Next, consider $\alpha$. In (A.5) let us disregard for the moment the dependence of $z_{0}$ and $\alpha$ on $\lambda$ and $\tau$, considering them instead to be free parameters. Then we may write the left side as a function. $F\left(\alpha, \lambda, \tau, z_{0}\right)$. When $\alpha=0, \tau=\infty$, and $z_{0}=z_{0}(\lambda)$, we have of course $h=0$, so that

$$
F\left(0, \lambda, \infty, z_{0}(\lambda)\right)=0
$$

Moreover,

$$
\frac{\partial F}{\partial \alpha}\left(0, \lambda, \tau, z_{0}\right)=\int_{-\infty}^{\infty} e^{c z}\left(V^{\prime}\left(z-z_{0}\right)\right)^{2} d z
$$

is nonzero and independent of $\tau$.

It can easily be checked that $F$ is a smooth function of its four variables, uniformly in a neighbourhood of $\left(0,0, \infty, z_{0}(0)\right)$. This follows by direct differentiation, using the boundedness of $v$ and its derivatives, and the exponential convergence of $V$ and its derivatives to their limits, uniformly in that neighbourhood. Therefore by the implicit function theorem, we may solve the equation $F\left(\alpha, \lambda, \tau, z_{0}\right)=0$ for $\alpha=\alpha\left(\tau ; \lambda, z_{0}\right)$ defined in a neighbourhood of $\left(\infty ; 0, z_{0}(0)\right)$, and $\alpha$ is a smooth function of its three variables in that neighbourhood. We now combine this with the fact that $z_{0}$ is a continuous function of $\lambda$ to obtain that $\alpha(\tau ; \lambda)$ is continuous, uniformly for $\lambda$ near 0 and $\tau$ near $\infty$. This implies that $\tau^{*}(\lambda)$ is bounded for $\lambda$ in that range. This establishes (iii).

This together with the continuity of $v$ in (iv), provides the continuity of $h$ in (iv). There remains only (v).

By [2], $h$ approaches 0 for each $\lambda$. Given any $\delta>0$, (1) let $T>\tau^{*}(0)$ be such that $|h(z, T ; 0)|<\frac{1}{2} \delta$. Then (2) let $\varepsilon_{1}$ be so small that $|h(z, T ; \lambda)-h(z, T ; 0)|<\frac{1}{2} \delta$ for $|\lambda|<\varepsilon_{1}$. This is possible by (iii) and (iv). Thus for $|\lambda|<\varepsilon_{1}(\delta)$, we have $\mid h\left(z, \tau(\delta) ;\left.\lambda\right|_{0}<\delta\right.$. This completes the proof.

Certain functions $w$ and $y$ figure into the proof of exponential convergence in $[2, \S 5]$. They are defined as follows. For a certain small enough positive number $\gamma, w(z, \tau)$ is set equal to $v(z, \tau)$ for $|z| \leqq \eta \tau$, and for larger $|z|, w$ attains the limit $h_{+}$or $h_{-}$smoothly within a $z$-interval of length $l$. We set

$$
\begin{gathered}
h^{*}(z, \tau)=w(z, \tau)-V\left(z-z_{0}-\alpha(\tau)\right), \\
y(z, \tau)=e^{\varepsilon z / 2} h^{*}(z, \tau),
\end{gathered}
$$

where $z_{0}$ (depending on $\phi$ ) and $\alpha$ are as defined above. In the context of Lemma A.1, 
these are all functions of $\lambda$ as well, and we must restrict $\tau>\tau^{*}(\lambda)$. We denote the $L_{2}$ norm over the real line by $\|\cdot\|$.

Lemma A.2. The norm $\|y(z, \tau ; \lambda)\|$ is continuous in $\lambda$, uniformly for $\tau>\tau^{*}(\lambda)$ in bounded intervals.

Proof. Let $\tau^{*}(\lambda)<\tau<\tau_{0}$. Let $Z$ be such that $w=h_{ \pm}(\lambda)$ for $|z|>Z$ in this $\tau$-interval for all $\lambda$, so that

$$
y(z, \tau ; \lambda)=-\exp \left(\frac{1}{2} c(\lambda) z\right)\left(V\left(z-z_{0}(\lambda)-\alpha(\tau ; \lambda) ; \lambda\right)-h_{ \pm}(\lambda)\right)
$$

for $|z|>Z, \tau<\tau_{0}, \lambda \in \Lambda$. Then $\int_{|z|>z} y^{2} d z$ depends continuously on $\lambda$, because of the uniform exponential convergence of $V$ to its limits at $\pm \infty$.

Also $h^{*}$, hence $y$, depends continuously on $\lambda$, uniformly for $\tau^{*}<\tau<\tau_{0},|z|<Z$, so that $\int_{|z|<z} y^{2} d z$ does as well. Combining these two establishes the lemma.

Lemma A.3. Let $\phi$ satisfy (A.2). Then there exist numbers $z_{1}<z_{2}, M$, and $v$, independent of $\phi$ and of $x_{0}$, such that

$$
V\left(z-z_{2}\right)-M e^{-v t}<v(z, \tau)<V\left(z-z_{1}\right)+M e^{-v \tau} .
$$

Proof. Let $z_{1}$ and $z_{2}$ be the numbers such that the solution $v(z, \tau)$ of (A.1) with $\phi$ replaced by $\phi_{*}(z)$ approaches $V\left(z-z_{2}\right)$ as $\tau \rightarrow \infty$, and the solution $\bar{v}(z, \tau)$ with initial datum $\phi^{*}$ approaches $V\left(z-z_{1}\right)$. By the maximum principle, the actual solution $v(z, \tau)$ lies between $\underline{v}$ and $\bar{v}$. The conclusion now follows directly from [2, Lemma 4.1] applied to $\underline{v}$ and to $\bar{v}$. The constants $M, z_{i}$, and $v$ are properties of the upper and lower solutions, so do not depend on $\phi$. Moreover, they do not depend on $x_{0}$ either, because the estimates used in the proof of [2, Lemma 4.1] depend only on the properties of $f\left(u, x_{0}\right)$, which are uniform in $x_{0}$, according to $\mathrm{H} 1-\mathrm{H} 3$.

Proof of Lemma 2.7. We recall that the proof of exponential convergence in $[2, \S 5]$ relied on the exponential decay of $\|y\|$ and of $\alpha(\tau)$ :

$$
\begin{gathered}
\|y\|<C e^{-v \varepsilon}, \\
|\alpha(\tau)|<C e^{-v \tau} .
\end{gathered}
$$

For our proof, then, it suffices to show that these two constants $C$ and $v$ do not depend on $\phi$ or $x_{0}$, under the conditions stated in Lemma 2.7. The constant $v$ certainly does not; it is clear from [2, §5] that it depends only on specific properties of the function $f$; by $\mathrm{H} 1-\mathrm{H} 3$, these properties are uniform in $x_{0}$. So any possible dependence would have to be in the constant $C$, which does in general depend on $\phi$. Also examining the proof in $[2, \S 5]$, it is clear that $C$ depends only on the time $T_{0}$ beyond which $\left|h^{*}\right|_{0}$ 
remains less than a certain sufficiently small number $\delta_{0}$ (independent of $\phi$ and $x_{0}$ ), and on the magnitudes of $\|y\|$ and $|\alpha|$ at that time $T_{0}$. Therefore for our proof, it suffices to show the existence of a number $T$, independent of $\phi$ or $x_{0}$, such that for $\tau>T$,

$$
\begin{gathered}
|h(z, \tau)|_{0}<\delta_{0}, \\
\|y\|_{0}<\delta_{0} \text { (say), }
\end{gathered}
$$

and

$$
|\alpha(\tau)|<\delta_{0}
$$

Assume this were not possible. Then there would be a sequence $\left\{\phi_{n}\right\}$ of functions satisfying (A.2) and of numbers $\left\{x_{n}\right\}$ such that for some sequence $T_{n}$ approaching $+\infty$, one of the inequalities (A.10-12) is violated for the solution corresponding to $\phi_{n}$ and $x_{n}$, for some $\tau>T_{n}$.

More specifically, let $v_{n}(z, \tau)$ be the solution of (A.1) with $c_{(0)}$ replaced by $c_{n} \equiv c\left(x_{n}\right), x_{0}$ by $x_{n}$, and $\phi$ by $\phi_{n}$. Then the corresponding functions $h_{n}, y_{n}, V_{n}, w_{n}$, and $\alpha_{n}$ have the obvious meaning. Placing subscripts " $n$ " on $h, y, \alpha$, and $T$ in (A.10)-(A.12), we suppose that at least one of them is violated for each $n$, for $\tau>T_{n}$, and that $T_{n} \rightarrow \infty$.

There is no loss of generality in assuming the $\phi_{n}$ 's to be smooth uniformly in $n$ and $z$; if they are not, then replace them by the functions $v_{n}\left(z, \tau_{1}\right)$ for any small positive $\tau_{1}$; the smoothing properties of the equation (A.1a) will establish the required equismoothness. In fact, for smooth enough $f$, standard estimates show that any given derivative is bounded in the uniform norm in terms of $\tau_{1}$ and $\left|\phi_{n}\right|_{0}$ alone. By this equicontinuity, there is a subsequence of the $\phi_{n}$ 's which converges to a limit function $\phi_{\infty}$ which also satisfies (A.2). We use the same symbol $\left\{\phi_{n}\right\}$ to denote this subsequence. This convergence is uniform for $z$ in bounded intervals. Again by equicontinuity, we may assume that there is a function $f_{\infty}(v)$ which is the uniform limit of the (same) subsequence of $f\left(v, x_{n}\right)$. Let $v_{\infty}(z, \tau)$ denote the solution with $f=f_{\infty}$ and initial datum $\phi_{\infty}$. In the notation $h_{n}, y_{n}, \alpha_{n}, z_{n}, V_{n}$ referred to above, we also allow $n=\infty$.

We now apply the results in $[2, \S 5]$ to $v_{\infty}$. They imply there is a number $T_{\infty}<\infty$ such that for $\tau>T_{\infty}$, (A.10)-(A.12) hold with subscript $n=\infty$, and with $\delta_{0}$ replaced by $\frac{1}{2} \delta_{0}$. For example,

$$
\left|\alpha_{\infty}(\tau)\right|_{0}<\frac{1}{2} \delta_{0} \text { for } \tau>T_{\infty} \text {. }
$$

Lemma A.1 is applicable; we set $\lambda_{n}=n^{-1}, \phi\left(\lambda_{n}\right)=\phi_{n}$, etc., so that results of the lemma about uniformity properties in $\lambda$ for $\lambda$ in a neighbourhood of 0 are now translated into uniformity properties in $n$ for $n$ sufficiently large. In particular, part (v) shows that (A.10) hold for $h=h_{n}$ with $n$ sufficiently large and $\tau>$ some $T_{1}$ independent of $n$. Therefore (A.10) is not violated for more than a finite number of $n$. This leaves (A.11) or (A.12).

The continuity statement (iii) in Lemma A.1 can be restated as follows: Given an $\varepsilon_{1}>0$, there exists a $\delta\left(\varepsilon_{1}\right)$ and a $\tau_{1}$ such that if 
(1) $\phi_{1}$ and $\phi_{2}$ are functions satisfying (A.2) and $\left|\phi_{1}-\phi_{2}\right|_{0}<\delta$, and

(2) $f_{1}(v)$ and $f_{2}(v)$ are bistable functions satisfying $\mathrm{H} 1-\mathrm{H} 3$ with $\left|f_{1}(v)-f_{2}(v)\right|<\delta$ for all $v$ in the range of interest,

then the corresponding functions $\alpha$ satisfy

$$
\left|\alpha_{1}(\tau)-\alpha_{2}(\tau)\right|<\varepsilon_{1}
$$

for all $\tau \geqq \tau_{1}$.

Now let $\varepsilon_{1}=\frac{1}{2} \delta_{0}$ and $\delta=\delta\left(\varepsilon_{1}\right)$. Choose $T_{1}$ and $Z_{1}$ so that $\left|v_{\infty}(z, \tau)-v_{n}(z, \tau)\right|<\frac{1}{2} \delta$ for $|z|>Z_{1}, \tau \geqq T_{1}$, and all $n$. This is possible by Lemma A.3. With no loss of generality, we may assume that $T_{1}>T^{*}\left(n^{-1}\right)$ for $n$ sufficiently large, and $T_{1}>T_{\infty}$. Then choose $N$ so that $\left|v_{\infty}-v_{n}\right|<\frac{1}{2} \delta$ for $|z|<Z_{1}, n>N$, and $\tau=T_{1}$. Thus for $n>N$.

$$
\left|v_{n}\left(z, T_{1}\right)-v_{\infty}\left(z, T_{1}\right)\right|<\delta
$$

so from (iii), $\left|\alpha_{n}(\tau)-\alpha_{\infty}(\tau)\right|<\varepsilon_{1}$ for all $\tau \geqq T_{1}+\tau_{1}\left(\varepsilon_{1}\right)$. Thus from (A.13),

$$
\left|\alpha_{n}(\tau)\right|<\delta_{0}
$$

for $n \geqq N, \tau \geqq T_{1}$. This shows that (A.12) holds for a number $T$ independent of $n$. This leaves (A.11).

Again, choose $Z_{2}$ such that

$$
\int_{|z|>Z_{2}} y_{n}^{2} d z<\frac{1}{8} \delta_{0} \text { for all } n, \tau=T_{\infty}
$$

Then take $N$ so that

$$
\int_{|z|<Z_{2}}\left(y_{n}^{2}-y_{\infty}^{2}\right)<\frac{1}{4} \delta_{0} \text { for } n>N, \tau=T_{\infty}
$$

It follows that

$$
\left\|y_{m}\right\|^{2}-\left\|y_{\infty}\right\|^{2}<\frac{1}{2} \delta_{0} \text { at } \tau=T_{\infty}
$$

From this and (A.11) with subscript $\infty$, we obtain that (A.11) holds as written with a fixed $T$ and $\tau>T$, but with a subscript $n$ attached by $y$. This contradicts our assumption, and we conclude that Lemma 2.7 is valid.

Acknowledgements. I am very grateful to L. A. Peletier for discussions on this topic. Supported by the National Science Foundation and by a British SERC Fellowship at Heriot-Watt University. 


\section{REFERENCES}

1. P. C. Fife and L. Hsiao, The generation and propagation of internal layers, Nonlinear Anal., TMA, 12 (1988), 19-41.

2. P. C. Fife and J. B. McLeod, The approacin of solutions of nonlinear diffusion equations to travelling front solutions, Arch. Rat. Mech. Anal. 65 (1977), 335-361.

3. P. C. FIFE, Long time behavior of solutions of bistable nonlinear diffusion equations, Arch. Rat. Mech. Anal. 70 (1979), 31-46.

4. P. C. Fife and L. A. Peletier, Clines induced by variable selection and migration, Proc. Roy. Soc. London B 214 (1981), 99-123.

5. P. C. Fife and L. A. Peletier, Unidirectional barriers to the propagation of fronts, in preparation.

6. R. A. Fisher, The advance of advantageous genes, Ann. of Eugenics 7 (1937), 355-369.

HERIOT-WATt UNIVERSITY

EDINBURGH

SCOTLAND
UNIVERSITY OF UTAH

Salt lake City UTAH, U.S.A. 